\title{
CATIE: Die Auswahl von Antipsychotika bei Schizophrenie
}

\begin{abstract}
Jürgen Fritze, Josef B. Aldenhoff, Frank Bergmann, Wolfgang Maier, Hans-Jürgen Möller
für die Deutsche Gesellschaft für Psychiatrie, Psychotherapie und Nervenheilkunde (DGPPN) und die Arbeitsgemeinschaft für Neuropsychopharmakologie und Pharmakopsychiatrie (AGNP)
\end{abstract}

psychoneuro 2005; 31 (10): 523-525
$\mathrm{D}$ as National Institute of Mental Health (NIMH) hat am 22.09.2005 die ersten Ergebnisse aus seinem „Clinical Antipsychotic Trials of Intervention Effectiveness" (CATIE) Projekt bezüglich der Wirksamkeit und Verträglichkeit der atypischen Antipsychotika Olanzapin, Risperidon, Quetiapin und Ziprasidon im doppelblinden, randomisierten Vergleich gegen das konventionelle Neuroleptikum Perphenazin publiziert (9). Dieses ist weltweit die erste Studie dieser Art, u.a. wegen ihrer Größe ( $n=1432)$, der Beobachtungsdauer (18 Monate) und der naturalistischen Bedingungen, die u.a. erlaubten, auch Patienten mit Komorbidität einzubeziehen. Sie wurde mit dem Ziel durchgeführt, die Vorteile in der alltäglichen Routineanwendung der modernen Antipsychotika gegenüber einem konventionellen Neuroleptikum zu prüfen.

Hauptzielvariable war die Zeit bis zum Abbruch der Studie i.w. wegen Wirkungsmangel oder Unverträglichkeit. Vorzeitiger Abbruch bedeutet dabei Beendigung der Behandlung unter Doppelblindbedingungen und Weiterbehandlung mit einem Antipsychotikum. Sekundärvariablen waren die spezifischen Absetzgründe sowie die mittels PANS (Positive and Negative Symptoms Scale) und CGI (Clinical Global Impression) ermittelte Wirksamkeit. Perphenazin, das in Deutschland nur $0,3 \%$ der verordneten Tagesdosen

Das Projekt CATIE des NIMH hat nur für das atypische Neuroleptikum Olanzapin, nicht aber für Risperidon, Quetiapin und Ziprasidon, begrenzte Überlegenheit gegenüber dem typischen Neuroleptikum Perphenazin gezeigt. Hauptzielvariable waren die Abbruchraten. Ziel der Therapie sind aber symptomatische Besserung, einschließlich kognitiver Störungen und Lebensqualität, die CATIE nicht erfasst hat, bei Vermeiden motorischer Nebenwirkungen, insbesondere von Spätdyskinesien, die unter atypischen Neuroleptika seltener sind. Das kann CATIE wegen eines methodischen Mangels nicht widerlegen. Entscheidend ist individualisierte Therapieanpassung.

der Neuroleptika ausmacht, wurde als Referenz gewählt, da es mit einem geringeren Risiko extrapyramidalmotorischer Nebenwirkungen verbunden zu sein schien als das am häufigsten verordnete und in den meisten Studien als Referenz eingesetzte Haloperidol. Clozapin blieb unberücksichtigt, da seine überlegene Wirksamkeit hinreichend belegt sei, Aripiprazol konnte nicht einbezogen werden, da zur Zeit des Studienbeginns noch nicht zugelassen.

Die Raten und die Zeiten bis zum Abbruch der Behandlung unter doppelblinden Bedingungen unterschieden sich zwischen den vier atypischen Neuroleptika und Perphenazin nicht signifikant. Olanzapin (64\%) war Quetiapin (82\%) und Risperidon (64\%) signifikant überlegen (Ziprasidon 79\%, Perphenazin 75\%). Jedoch nahmen unter Olanzapin 30\% der Patienten mehr als 7\% Gewicht zu mit Erhöhungen von HbA1C, Cholesterin und Triglyzeriden. Nominal brachen wegen extrapyramidalmotorischer Nebenwirkungen mehr Patienten Perphenazin (8\%) ab als die atypischen Neuroleptika (<4\%).
Die vier atypischen Neuroleptika wiesen also gegenüber dem Perphenazin nur marginale Vorteile auf, am deutlichsten Olanzapin. Diese Ergebnisse können zu der Schlussfolgerung Anlass geben, den deutlich höheren (ca. 10-fach) Tagesbehandlungskosten der vier atypischen Neuroleptika stehe kein entsprechender Zusatznutzen gegenüber. Hier ist aber Vorsicht angezeigt. Die Autoren wie auch das Editorial enthalten sich mit Bedacht einer solchen Schlussfolgerung.

Methodisch ist ein bedeutsamer Mangel, dass Patienten mit vorbestehender tardiver Dyskinesie selektiv nur von der Behandlung mit Perphenazin ausgeschlossen wurden (12). Im Vergleich von geplanter zu tatsächlicher Rekrutierung darf geschätzt werden, dass dies rund 80 Patienten (also 25\% der geplanten) betraf. Inzidenz und Prävalenz von tardiver Dyskinesie und akuten extrapyramidalmotorischen Nebenwirkungen hängen eng zusammen, letztere sind - neben anderen Risikofaktoren - Prädiktoren für tardive Dyskinesie (Barnes \& McPhillips 1998). Damit bedeutet das Aus- 
schlusskriterium nur im Perphenazin-Arm von CATIE potentiell eine erhebliche Verfälschung der Befunde zur extrapyramidalmotorischen Verträglichkeit zugunsten von Perphenazin, indem zu postulieren ist, dass in den vier anderen Studienarmen Patienten mit Risiko für extrapyramidalmotorische Nebenwirkungen mit 25\% überrepräsentiert waren.

Die Vorteile wie auch die Nachteile unter Olanzapin können damit zusammen hängen, dass CATIE (nur) für Olanzapin eine Dosierung (maximal $30 \mathrm{mg} /$ Tag) oberhalb der zugelassenen Höchstdosis (20 mg) erlaubte, so dass die tatsächliche Dosis mit im Mittel $20 \mathrm{mg} /$ Tag relativ zu den anderen geprüften Neuroleptika hoch lag.

Die Raten des Abbruchs der Behandlung unter doppelblinden Bedingungen sind auf den ersten Blick erschreckend hoch. Hier gilt es zu berücksichtigen: a) Es wurden keine gezielten Maßnahmen wie Psychoedukation (11) ergriffen, um die Halterate zu erhöhen. Weiden \& Olfson (13) ermittelten für Haloperidol und Fluphenazin in der Routineanwendung eine mittlere Abbruchrate (Noncompliance) von 7,6\% pro Monat. b) Lieberman et al. (9) konzedieren selbst, dass der Kontext einer verblindeten Studie die Abbruchrate erhöht haben könnte. c) Die Studie setzt Patienten voraus, die bereit sind, ihre bisherige Behandlung abzubrechen, um an einer Doppelblindstudie mit vermutlich anderer als ihrer bisherigen Medikation teilzunehmen; diese Voraussetzung ist in einer repräsentativen Stichprobe von Patienten mit langfristiger Antipsychotikatherapie nicht erreichbar; die Bereitschaft zur Studienteilnahme dürfte mit einem eher ungünstigen Verlauf und geringerem Therapieansprechen verbunden sein, der auch in der Studie durch erhöhte Abbruchraten in Erscheinung tritt.

Therapieziel bei der langfristigen, ambulanten Anwendung von Antipsychotika unter naturalistischen Bedingungen ist nicht die Haltequote, die in der Studie das Hauptkriterium darstellt. Unter naturalistischen Bedingungen geht es vielmehr primär um die Besserung der
Fähigkeit zur Nutzung der Lebenschancen im Berufs- und Privatleben, um die Steigerung von Lebensqualität und Lebenszufriedenheit. Dieses primäre, praktische Therapieziel ist in der „Abbruchrate“ in einer Doppelblindstudie nicht abgebildet. Diese praktisch besonders bedeutsamen Parameter wurden nicht gemessen, ebenso wenig wie die hierzu beitragende kognitive Leistungsfähigkeit und Medikamentenpräferenz der therapieerfahrenen Patienten. Diese primär naturalistischen Therapieziele werden meist durch eine Umstellung der Antipsychotika auch gar nicht gefährdet. So ist die Aussagekraft dieser als ,naturalistisch“ geplanten Studie durch die mangelnde Praxisrelevanz des Hauptzielkriteriums erheblich eingeengt. Keinesfalls kann das berichtete Hauptergebnis der Studie Grundlage gesundheitspolitischer Entscheidungen werden.

Die bisher verfügbaren Evidenzen sprechen für eine höhere Effektivität von Atypika im Hinblick auf alltagsrelevante Funktionen: Keefe et al. (7) fanden in ihrer Meta-Analyse von 15 Studien - nur 5 davon verblindet - Evidenz für eine überlegene Besserung kognitiver Funktionen unter atypischen Neuroleptika. Miyamoto et al. (10) beschreiben in ihrem qualitativen Review für spezifische kognitive Funktionen (z.B. verbale Flüssigkeit, Feinmotorik) eine Überlegenheit atypischer Neuroleptika bei allerdings weiterem Forschungsbedarf. Awad \& Voruganti (2) sehen in ihrem qualitativen Review trotz methodischer Schwächen der Studien und ihrer begrenzten Anzahl (überwiegend bezüglich Risperidon, Olanzapin and Clozapin) einen „deutlichen Trend in Richtung günstigerer Wirkungen der Antipsychotika der zweiten Generation auf die Lebensqualität". Lambert \& Naber (8) sehen erhebliche Evidenzen für größere Lebensqualität und subjektives Wohlbefinden unter atypischen als unter konventionellen Neuroleptika.

\section{Was bedeutet das für die Praxis?}

Hier kann nur den Autoren und dem Editorial von Freedman gefolgt werden: Das Neuroleptikum ist nach Verträglichkeit und Präferenzen des Patienten den individuellen Bedingungen angepasst auszuwählen. CATIE hat keine differentielle, individualisierte Indikation erlauben können, sondern randomisiert. Der mehr als doppelt so hohe Anteil atypischer Neuroleptika an den Gesamtverordnungen der Neuroleptika in den USA wie in Deutschland (2003: atypische 31\%; 4) legt nahe, dass die Indikation in Deutschland ohnehin differenzierter gestellt wird. Eine bedeutsame Rolle spielt dabei auch das Risiko tardiver Dyskinesien, das unter konventionellen Neuroleptika - und in CATIE auch unter Perphenazin - höher als unter atypischen Neuroleptika ist $(5,6)$. Die jährliche Inzidenz wird für typische Neuroleptika auf ca. 5\% gegenüber ca. $1 \%$ für atypische geschätzt (6). Adams et al. (1) fanden keine Hinweise, dass sich die verschiedenen typischen Neuroleptika (u.a. Perphenazin und Haloperidol) in ihrem Risiko unterscheiden. Die Ergebnisse von CATIE können wegen des möglichen, oben beschriebenen Selektionsartefaktes zugunsten Perphenazin nicht widerlegen, dass die atypischen Neuroleptika eine bessere extrapyramidalmotorische Verträglichkeit und ein geringeres Risiko der Entwicklung tardiver Dyskinesien aufweisen. Das ist klinisch relevant. Hierüber ist der einzelne Patient aufzuklären. Hieran wird der aufgeklärte Patient seine Präferenz orientieren.

Vor diesem Hintergrund gibt CATIE keinen Anlass, die Priorisierung atypischer Neuroleptika in den Leitlinien (z.B. der World Federation of Societies of Biological Psychiatry, WFSBP, und der DGPPN) zu modifizieren.

Insgesamt ist unverkennbar, dass es weiterer Forschung bedarf, insbesondere auf der Suche nach noch besseren Antipsychotika.

\section{CATIE: choice of antipsychotics in schizophrenia}

The CATIE project of the NIMH has revealed moderately superior effectiveness of atypical neuroleptics only for olanzapine, but not risperidone, quetiapine and ziprasidone, compared to 
the typical perphenazine. Primary outcome measure was the discontinuation for any cause. The aim of routine treatment, however, is symptomatic improvement including cognitive functioning and quality of life which were not assessed in CATIE, and the avoidance of motor side effects, especially tardive dyskinesia which occurs less frequently under atypical neuroleptics. Due to a methodological flaw CATIE cannot disprove this. Individualized adaption of treatment is decisive.

\section{Key words}

CATIE - atypical neuroleptics schizophrenia

\section{Literatur:}

1. Adams CE, Fenton MKP, Quraishi S, David AS. Systematic meta-review of depot antipsychotic drugs for people with schizophrenia. Br J Psychiatry 2001; 179: 290-299 2. Awad AG, Voruganti LNP: New Antipsychotics, Compliance, Quality of Life, and Subjective Tolerability - Are Patients Better
Off? Can J Psychiatry 2004; 49: 297-302

3. Barnes TR, McPhillips MA. Novel antipsychotics, extrapyramidal side effects and tardive dyskinesia. Int Clin Psychopharmacol 1998; 13 Suppl 3: 49-57

4. Fritze J. Psychopharmaka-Verordnungen: Ergebnisse und Kommentare zum Arzneiverordnungsreport 2004. Psychoneuro 2005; 31: 46-52

5. Jeste DV. Tardive dyskinesia rates with atypical antipsychotics in older adults. J Clin Psychiatry 2004; 65: Suppl 9: 21-24

6. Kane JM: Tardive dyskinesia rates with atypical antipsychotics in adults: prevalence and incidence. J Clin Psychiatry 2004; 65 (Suppl 9): 16-20

7. Keefe RS, Silva SG, Perkins DO, Lieberman JA. The effects of atypical antipsychotic drugs on neurocognitive impairment in schizophrenia: a review and meta-analysis. Schizophr Bull 1999; 25: 201-222

8. Lambert M, Naber D: Current issues in schizophrenia: overview of patient acceptability, functioning capacity and quality of life. CNS Drugs 2004; 18: Suppl 2: 5-17

9. Lieberman JA, Stroup TS, McEvoy JP, Swartz MS, Rosenheck RA, Perkins DO, Keefe RSE, Davis SM, Davis CE, Lebowitz BD, Severe J, Hsiao JK. Effectiveness of Antipsychotic Drugs in Patients with Chronic Schizophrenia. New England Journal of Medicine
2005; 353: 1209-1223

10. MiyamotoS, Duncan GE, Marx CE, Lieberman JA. Treatments for schizophrenia: a critical review of pharmacology and mechanisms of action of antipsychotic drugs. Molecular Psychiatry 2005; 10: 79-104

11. Pekkala E, Merinder L. Psychoeducation for schizophrenia. Cochrane Database Syst Rev 2002

12. Stroup TS, McEvoy JP, Swartz MS, Byerly M], Glick ID, Canive JM, McGee MF, Simpson GM, Stevens MC, Lieberman JA. The National Institute of Mental Health Clinical Antipsychotic Trials of Intervention Effectiveness (CATIE) project: schizophrenia trial design and protocol development. Schizophr Bull 2003; 29: 15-31

13. Weiden PJ, Olfson M: Cost of relapse in schizophrenia. Schizophr Bull 1995; 21: 419-429

\section{Korrespondenzadresse:}

Prof. Dr. med. Jürgen Fritze

Gesundheitspolitischer Sprecher

Deutsche Gesellschaft für Psychiatrie,

Psychotherapie \& Nervenheilkunde

(DGPPN)

Asternweg 65

50259 Pulheim 